\title{
UCRL-JRNL-226377
}

LAWRENCE LIVERMORE NATIONAL LABORATORY

\section{Multilayer High-Gradient Insulators}

J. R. Harris, R. M. Anaya, D. Blackfield, Y. -J. Chen, S. Falabella, S. Hawkins, C. Holmes, A. C. Paul, S. Sampayan, D. M. Sanders, J. A. Watson, G. J. Caporaso, M. Krogh

November 28, 2006

IEEE Transactions on Dielectrics and Electrical Insulation 
This document was prepared as an account of work sponsored by an agency of the United States Government. Neither the United States Government nor the University of California nor any of their employees, makes any warranty, express or implied, or assumes any legal liability or responsibility for the accuracy, completeness, or usefulness of any information, apparatus, product, or process disclosed, or represents that its use would not infringe privately owned rights. Reference herein to any specific commercial product, process, or service by trade name, trademark, manufacturer, or otherwise, does not necessarily constitute or imply its endorsement, recommendation, or favoring by the United States Government or the University of California. The views and opinions of authors expressed herein do not necessarily state or reflect those of the United States Government or the University of California, and shall not be used for advertising or product endorsement purposes. 


\title{
Multilayer High-Gradient Insulators
}

\section{J.R. Harris, R.M. Anaya, D. Blackfield, Y.-J. Chen, S. Falabella, S. Hawkins, C. Holmes, A.C. Paul, S. Sampayan, D.M. Sanders, J.A. Watson, and G.J. Caporaso}

\author{
Lawrence Livermore National Laboratory \\ Livermore, CA 94551 \\ M. Krogh \\ University of Missouri \\ Rolla, MO 65401
}

\begin{abstract}
High voltage systems operated in vacuum require insulating materials to maintain spacing between conductors held at different potentials, and may be used to maintain a nonconductive vacuum boundary. Traditional vacuum insulators generally consist of a single material, but insulating structures composed of alternating layers of dielectric and metal can also be built. These "High-Gradient Insulators" have been experimentally shown to withstand higher voltage gradients than comparable conventional insulators. As a result, they have application to a wide range of highvoltage vacuum systems where compact size is important. This paper describes ongoing research on these structures, as well as the current theoretical understanding driving this work.
\end{abstract}

Index Terms — Insulator testing, flashover, pulse power systems, accelerators.

\section{INTRODUCTION}

VACUUM insulators are widely used in high voltage systems to maintain physical spacing of components and provide a nonconductive vacuum boundary [1]. They tend to fail due to surface flashover, rather than breakdown through the insulator bulk. While there remains much debate regarding the exact mechanisms responsible for surface flashover, it is widely believed to be initiated by a secondary electron avalanche occurring along the insulator-vacuum boundary [2,3]. This liberates gas which was previously adsorbed by the insulator surface, and it is this gas which breaks down to produce the surface flashover. Insulator surface flashover sets limits on the voltage gradients that can be applied in a system, and therefore on the compactness of a given high voltage vacuum device.

A number of approaches have been used, with varying success, to increase the surface flashover strength of vacuum insulators. These include the use of surface coatings to reduce secondary electron yield (especially in the case of multipactoring [4]), magnetic insulation [5], angled insulator geometry [3], and physical shielding of the electrode from charged-particle beams.

A different approach, known as the "High Gradient Insulator" (HGI), is currently under development. HGIs, also known as microstacks, are insulating structures consisting of thin, alternating metal and dielectric layers. These structures have exhibited surface flashover strengths that are up to four times greater than conventional, straight-wall insulators [6]. Operation above $15 \mathrm{MV} / \mathrm{m}$ gradients is routine, and with sufficiently short pulses, gradients of $100 \mathrm{MV} / \mathrm{m}$ have been demonstrated [7]. HGIs are tolerant of being placed in direct view of high-current electron [8,9] and ion [10] beams. In addition, they have microwave properties that are useful for accelerators [11]. HGIs are a key enabling technology for a series of new, innovative accelerator concepts, including improved induction cells [12,13], novel optically-triggered high-voltage switches [14], and dielectric-wall accelerators [9,15-18].

In this paper, we will review the theories that have guided previous work on HGIs and describe the research currently being conducted on these structures at Lawrence Livermore National Laboratory.

\section{THEORIES OF OPERATION}

It is well known that shorter vacuum insulators are able to withstand higher gradients. In order to span a long distance but still take advantage of this effect, an insulating structure can be assembled from a series of very short insulators, separated by conducting layers to form an HGI. This concept was recognized and investigated by I. Smith [19], and later by E. Gray [6]. More recently, research has been driven by two models developed by researchers in the United States and 
Israel. Both of these models focus on interrupting the secondary electron avalanche believed to precipitate surface flashover. However, they propose to do this in different ways, which results in different insulator geometries. The first model, developed by J.M. Elizondo and his collaborators, relies on physical interception of avalanching electrons by the HGI metal layers [20]. In this model, the maximum thickness of the dielectric layers must be less than the expected electron range, so that electrons are intercepted by a metal layer before they can collide with the dielectric and produce secondaries. Electron trajectories will not generally be parallel to the HGI surface. To intercept electrons launched with nonzero initial angles, the metal layers may protrude to form "fins," with their height above the dielectric surface determined by the expected maximum height of the electron trajectories [21]. Additional design criteria may include considerations of the pulse length and expected residue gasses in the system [20], generation of photoelectrons by photons produced in streamer tips [21], and the maximum capacity of the HGI structure to absorb charge before failure [21] or re-emission [22].

HGIs designed following these guidelines have dielectric layers which are on the order of $500 \mu \mathrm{m}$ thick [20], interspersed with much thinner metal layers that protrude beyond the dielectric; to date, most HGIs have been fabricated more or less along these lines. Experience has shown that, in addition to being difficult to fabricate and maintain, HGIs with protruding vanes did not perform much differently than those without protruding vanes [23], and so most recently-built HGIs have used metal layers that are nominally flush with the dielectric surface. Finally, the thin metal layers typical of these designs are susceptible to vaporization under vacuum flashover conditions due to their small thermal mass, and this metal has been observed to be deposited on the surface of the HGI [7].

HGIs designed following these procedures show excellent voltage-holding capability, which generally improves with decreasing pulse length and decreasing dielectric layer thickness [7].

More recently, a different operating mechanism for HGIs has been proposed by J.G. Leopold and his collaborators [24]. This model takes advantage of distortions in the potential distribution near the HGI surface resulting from the presence of the metal layers (Fig. 1). These distortions produce a periodic electric field perpendicular to the HGI surface, and function like the alternating gradient focusing systems used for charged particle beam transport $[25,26]$. If the geometry is chosen correctly, these fields may be able to interrupt a secondary electron emission avalanche by sweeping electrons away from the HGI surface. The structure begins with a halflayer of metal, which causes electrons generated from the cathode to be swept away from the HGI surface. In addition, electrons generated from the metal-dielectric interfaces along the HGI are more problematic if thicker dielectric layers and thinner metal layers are used, as these electrons are more likely to strike the HGI surface on regions of the dielectric where the surface electric field allows any secondaries to escape. For structures where the insulator thickness is less than three times the metal layer thickness, Leopold observed HGI performance to be much better than an equivalent solid dielectric insulator; for structures where the insulator thickness is more than three times the metal layer thickness, they observed HGI performance to be slightly worse than an equivalent solid dielectric insulator. Under this model, decreasing the HGI period results in a more gentle sweeping of electrons away from the HGI surface.

HGIs designed following these procedures have generally used thicker layers (typically a few millimeters) than those designed following Elizondo's protocol, but were also operated at lower overall gradients ( $\sim \mathrm{MV} / \mathrm{m})$.

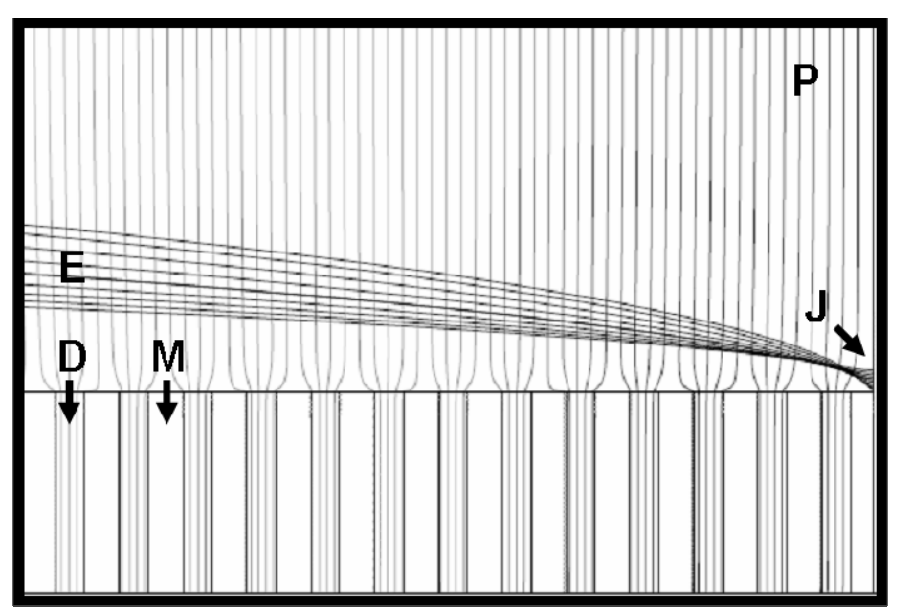

Fig. 1. Trak Simulation of Leopold-type HGI. Insulator structure consists of alternating layers of metal (M) and dielectric (D) of equal thickness. Far from the surface, potential lines (P) are uniformly spaced, but are distorted near the surface, creating an alternating electric field normal to the surface. The initial layer consists of a half-layer of metal, so that electrons produced from the HGI-cathode-vacuum interface (J) will be launched on trajectories (E) carrying them away from the HGI surface (After [24]).

\section{CURRENT RESEARCH}

At Lawrence Livermore, we are engaged in a comprehensive research program to investigate all aspects of HGI design and operation. In this section, we will describe our process for fabricating HGIs, our facilities, and some of our ongoing experimental efforts.

\subsection{HGI Fabrication and Preparation}

The HGIs that have been tested at Livermore have generally used Rexolite or Cirlex for the dielectric layers, and stainless steel for the metal layers. These structures are prepared by hot pressing appropriate thicknesses of stainless and polymer sheets to the desired laminate thickness. These laminates are subsequently machined and polished to the desired dimensions and surface finish.

\subsection{Small Sample Test Stand}

We currently use two systems for testing HGIs. For small samples, we have a dedicated test stand. At the heart of this test stand is a 16-stage Marx generator. Each stage can be 


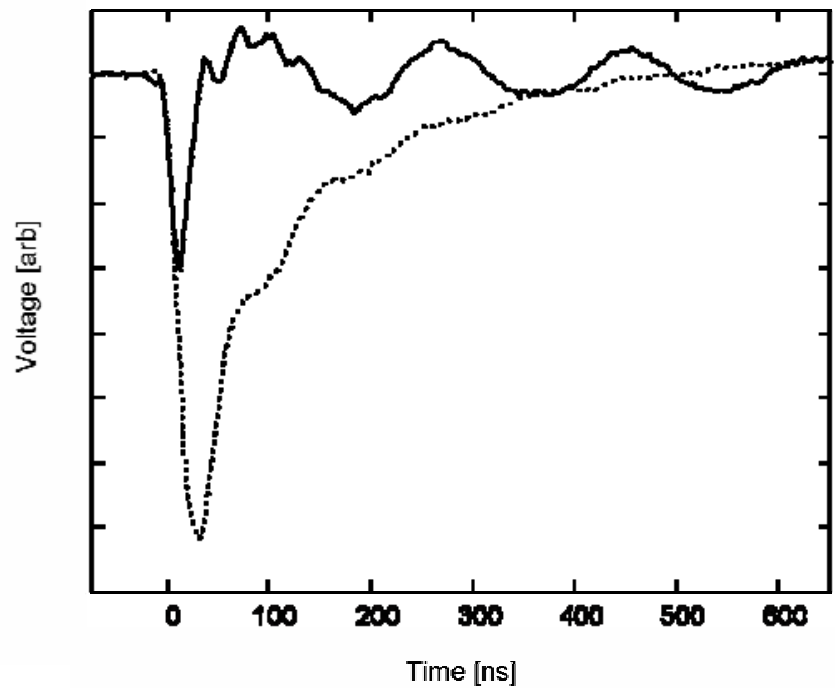

Fig. 2. Voltage traces recorded on the small test stand, showing normal waveform (dot) and waveform during HGI surface flashover (solid). Both waveforms were taken with the Marx charged to the same voltage, but during the flashover a peak voltage of only $97 \mathrm{kV}$ was developed, compared to 229 $\mathrm{kV}$ in the other case.

charged to $25 \mathrm{kV}$ and has a nominal capacitance of $2.8 \mathrm{nF}$. Conventional spark gaps are used for switching, with a trigatron used to trigger the first stage. To increase the dynamic range of the Marx, it is enclosed in a pressure vessel which can be operated up to $50 \mathrm{psi}$, and is normally used with ultra zero air. At low charging voltages, the Marx consistently produces an output voltage that is 12.3 times the charging voltage; it is less efficient at higher charging voltages, with a peak output of about $260 \mathrm{kV}$. A fluid resistor connected from the high voltage output of the Marx to ground serves to limit the pulse length to about 100 ns FWHM (Fig. 2). Prior to HGI tests with this system, the Marx operation was carefully characterized. The shot-to-shot variation in the output voltage is typically less than about $1.3 \%$. At a given pressure, there is a minimum charging voltage below which the Marx spark gaps will not trigger, and a maximum charging voltage above which the spark gaps self-break. For a given pressure, shotto-shot voltage reproducibility is better at higher voltages, while the Marx voltage gain is generally highest when operating near the middle of its working voltage range. For purposes of HGI testing, the data from these characterization tests was used to establish a set of operating points which provide the most reliable and reproducible operation of the Marx.

The insulator test chamber is operated at a pressure of about $2 \times 10^{-7}$ Torr, and contains a pair of electrodes used to apply voltage to the HGI samples. The electrodes are radiused to prevent field enhancements, and made of 304 stainless steel polished to approximately $6 \mu$ in RMS surface roughness. The spacing is adjustable to accept HGI samples of varying length. An upper limit on the useable voltage gradient is set by the intrinsic vacuum arc strength of the electrodes. One pair was tested and failed at a peak applied gradient of $42 \mathrm{MV} / \mathrm{m}$ with a 5 mm gap.
Test stand diagnostics include gated and open-shutter cameras, and capacitive and resistive voltage monitors. In addition, HGI samples are inspected before and after testing using a long focus zoom microscope outfitted with a video camera and image capture software. A computer controlled stepping motor arrangement is used to provide a complete record of the HGI surface.

\subsection{Small Sample Test Results}

The small HGI samples tested so far consist of stainless steel and Rexolite, are $2.54 \mathrm{~cm}$ in diameter, and vary between $2.65 \mathrm{~mm}$ and $15.18 \mathrm{~mm}$ in height. In some tests, these samples withstood gradients in excess of $21 \mathrm{MV} / \mathrm{m}$. Tests conducted to date focused on the effects of conditioning and the damage resulting from surface flashover. Tests were also carried out to compare the competing HGI geometries discussed in Section 2. In addition, we made observations regarding the speed of the breakdown events. Table 1 gives key parameters for insulators mentioned in this article.

Table 1. Summary of Insulators.

\begin{tabular}{|c|c|c|c|c|}
\hline HGI & $\begin{array}{c}\text { Peak Grad. } \\
(\mathbf{M V} / \mathbf{m})^{\mathbf{1}}\end{array}$ & Metal & Dielectric & $\begin{array}{c}\text { Length } \\
(\mathbf{m m})\end{array}$ \\
\hline R010 & $21.1+^{2}$ & $\begin{array}{c}0.013 \mathrm{~mm} \\
\text { stainless steel }\end{array}$ & $\begin{array}{c}0.254 \mathrm{~mm} \\
\text { Rexolite }\end{array}$ & 12.31 \\
\hline R011 & 10 & $\begin{array}{c}0.305 \mathrm{~mm} \\
\text { stainless steel }\end{array}$ & $\begin{array}{c}0.254 \mathrm{~mm} \\
\text { Rexolite }\end{array}$ & 12.36 \\
\hline R171 & $13^{3}$ & $\begin{array}{c}0.013 \mathrm{~mm} \\
\text { stainless steel }\end{array}$ & $\begin{array}{c}0.508 \mathrm{~mm} \\
\text { Rexolite }\end{array}$ & 10.15 \\
\hline R173 & 20.4 & $\begin{array}{c}0.013 \mathrm{~mm} \\
\text { stainless steel }\end{array}$ & $\begin{array}{c}0.508 \mathrm{~mm} \\
\text { Rexolite }\end{array}$ & 10.15 \\
\hline R201 & N/A & $\begin{array}{c}0.013 \mathrm{~mm} \\
\text { stainless steel }\end{array}$ & $\begin{array}{c}1.27 \mathrm{~mm} \\
\text { Rexolite }\end{array}$ & 2.65 \\
\hline Rex. Slug & 16.6 & N/A & Rexolite & 12 \\
\hline $\begin{array}{c}\text { Large } \\
\text { HGI }\end{array}$ & $\sim 14$ & $\begin{array}{c}\text { 0.013 mm } \\
\text { stainless steel }\end{array}$ & $\begin{array}{c}0.254 \mathrm{~mm} \\
\text { Kapton }\end{array}$ & 35 \\
\hline
\end{tabular}

${ }^{1}$ Highest peak gradient supported without flashover

${ }^{2}$ Strength exceeded voltage capability of test stand.

${ }^{3}$ Strength after being damaged by 223 flashover events.

${ }^{4}$ Flashover at $7 \mathrm{MV} / \mathrm{m}$ on first shot, no subsequent shots.

In a typical testing and conditioning sequence, progressively higher voltage pulses are applied across the sample. In all cases, one or more flashover events occur at lower gradients, after which the HGI can be operated at higher gradients. The character of the observed flashovers changes as the test progresses. Initial flashover events are dominated by large regions of luminous gas (Fig. 3, upper left image), while later flashover events are dominated by narrow, vertical discharges between adjacent metal layers (Fig. 4). Although the testing protocol is usually structured so that the earlier flashovers coincide with lower gradients, observed changes in the character of the discharge are probably a result of surface conditioning and accumulated damage rather than a change in gradient per se. For example, Fig. 3 shows three images of the same HGI undergoing surface flashover at different points in the initial phase of its testing sequence. The Marx operating conditions remained approximately unchanged during this sequence. As the testing progresses, the flashover 
pattern becomes less pronounced, with smaller discharges occurring at
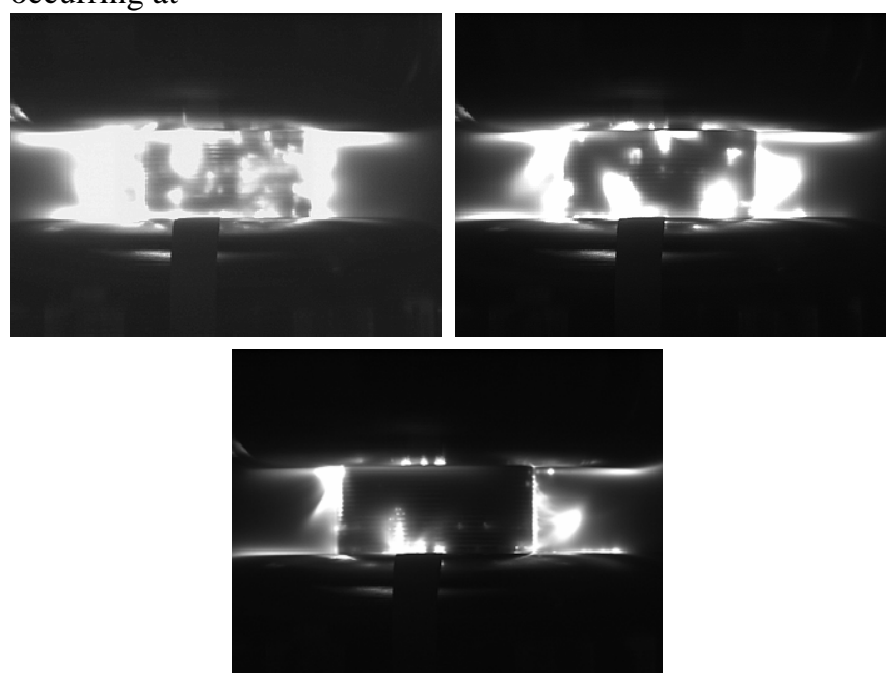

Fig. 3. HGI "R171." Surface flashover events at approximately $8 \mathrm{MV} / \mathrm{m}$, shots number 1 (top left), 5 (top right), and 15 (bottom). Note change in flashover pattern. Sample height is $10.15 \mathrm{~mm}$. (All flashover images presented show the cathode below the sample and the anode above the sample.)

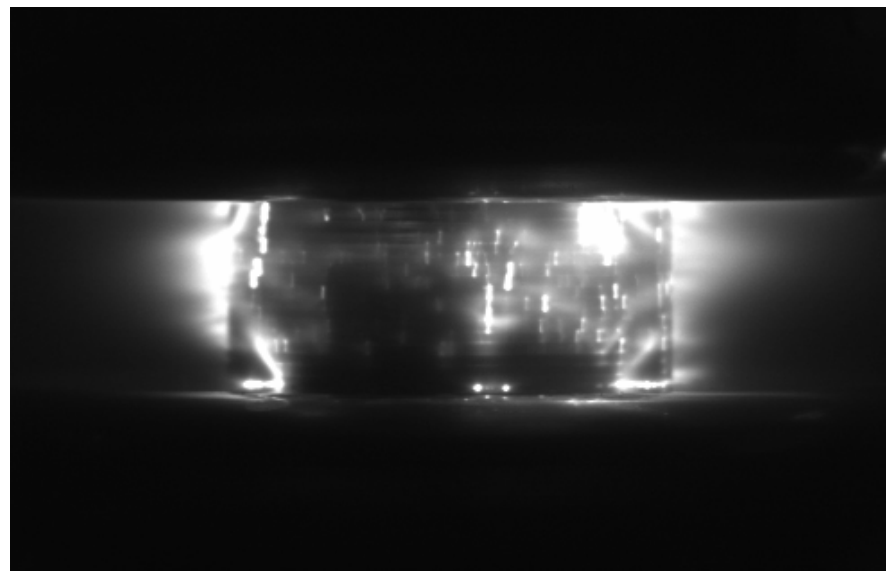

Fig. 4 HGI "R173." Surface flashover at $19.7 \mathrm{MV} / \mathrm{m}$. Sample height is 10.15 $\mathrm{mm}$. Note that this HGI was subjected to different testing procedures than the HGI shown in Figs. 4 and 7; however, it was produced in the same lot and is of identical design and construction. The flashover pattern shown here resulted in the deposition of stainless steel traces shown in Fig. 6.

fewer locations on the insulator surface. This is probably due to a progressive removal of gas and debris from the HGI surface by the flashover events. The observed changes in the flashover pattern also coincided with a subtle improvement in the voltage-holding capability of the insulator.

Despite this initial conditioning effect, additional flashovers eventually result in an accumulation of damage to the HGI surface. This damage depends on the applied gradient as well as the testing history of the insulator, and changes in the damage mechanism coincide with changes in the observed flashover pattern. At lower to intermediate gradients, damage primarily consists of discoloration of the Rexolite (Fig. 5).
This discoloration displays a spotty or wispy pattern, consistent with surface heating due to the diffuse discharge

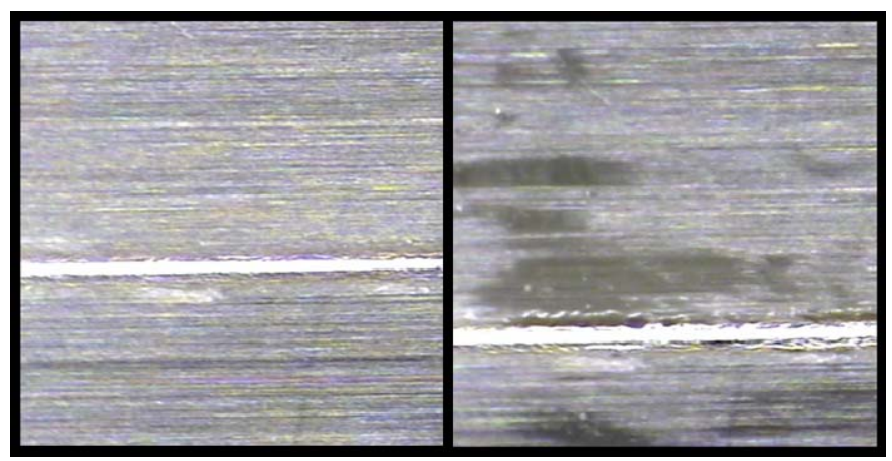

Fig. 5. HGI "R201." Surface before (left) and after (right) one flashover event at $7 \mathrm{MV} / \mathrm{m}$. The horizontal white line in each image is a $0.013 \mathrm{~mm}$ thick metal layer. Both images were taken at the same location on the HGI surface.

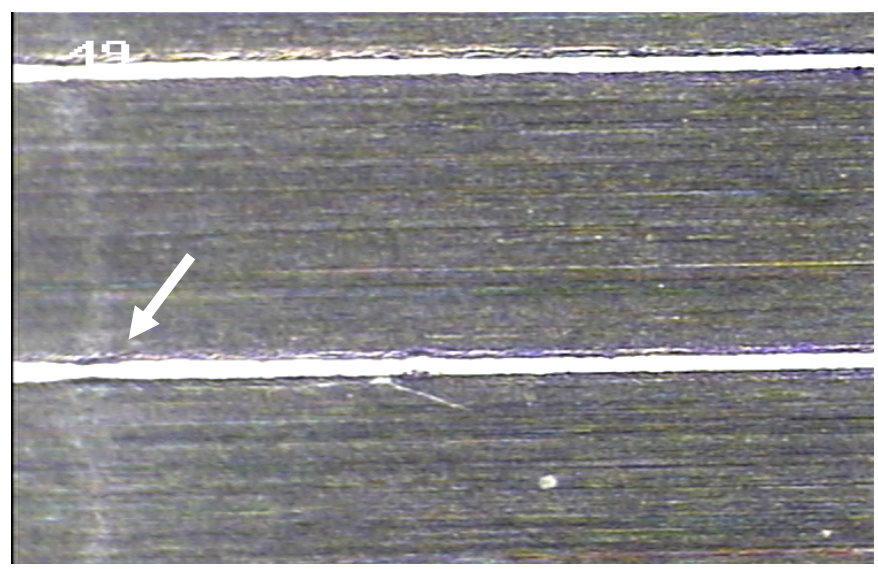

Fig. 6. HGI "R173." Erosion of metal layers and deposition of stainless traces. Metal layer thickness is $0.013 \mathrm{~mm}$.

pattern shown in Fig. 2. At higher gradients, damage is initially dominated by erosion of the thin metal layers, and deposition of white, vertical streaks on the Rexolite (Fig. 6). Energy-dispersive $\mathrm{x}$-ray measurements have confirmed that the

streaks consist of stainless steel. This pattern of damage is consistent with the small, vertical streaks seen in the flashover pattern shown in Fig. 4. This effect was fist observed after a series of about twenty flashover events with peak gradients near the upper operating limit of the HGI.

Most tests conducted so far have included a gradual increase in the applied gradient in order to achieve a conditioning effect, minimize the number of flashovers, and maximize the ultimate tested strength of the insulator. However, HGIs which are used in actual systems are likely to experience a large number of flashover events over an extended period of time. To investigate the behavior of HGIs under conditions of this type, an HGI was subjected to a total of 223 flashover events; some of these events are shown in Figs. 3 and 7. Of the 223 flashover events, 200 were due to shots taken at nearly full charging voltage and 23 were due to additional shots taken at lower charging voltage. No low-charging- 
voltage conditioning was attempted prior to testing. Serious damage

occurred, including discoloration and ablation of both the metal and dielectric layers over significant portions of the HGI
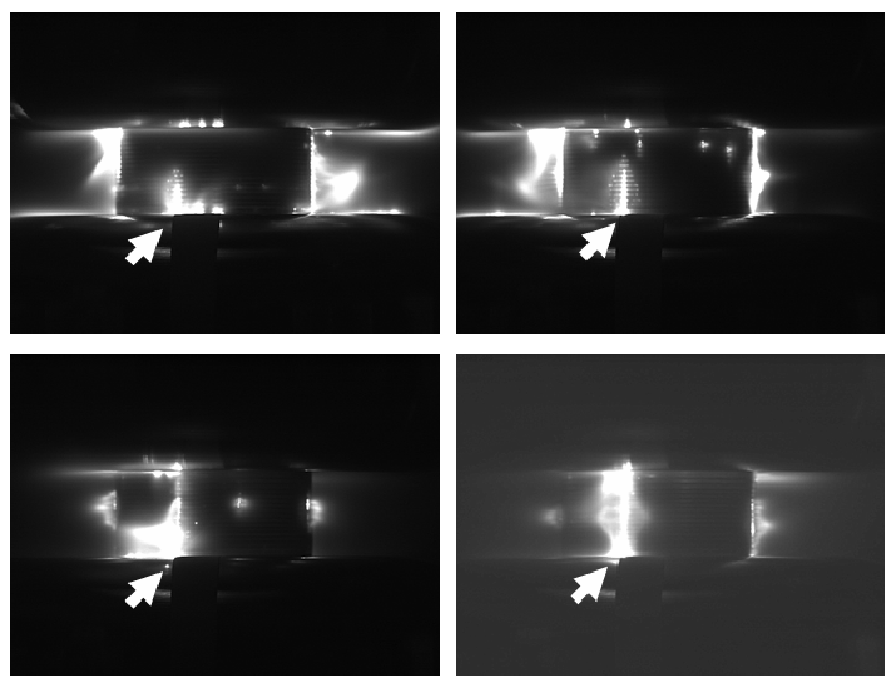

Fig. 7. HGI "R171." Shots number 15 (top left), 57 (top right), 182 (bottom left), and 198 (bottom right). Note the worsening of the flashover at the location indicated by the arrow.

surface. However, on the 224th shot the HGI held $13 \mathrm{MV} / \mathrm{m}$ without flashover. This is $64 \%$ of its expected strength based on previous tests with an identical part which was subjected to a typical conditioning and testing routine.

The HGI from Figs. 3 and 7 is shown in Figs. 8 and 9 after the completion of testing. Microphotographs taken of the HGI surface showed the presence of stainless steel streaks in much higher numbers, and covering a larger surface area, than was seen in the previous tests. However, the serious damage resulting from this test went beyond that seen on previous tests, and was reflected in the observed flashover images and voltage-holding ability of the sample. Fig. 7 shows four representative flashovers taken from the second part of the same testing sequence. This figure shows a slow worsening of the flashover as more shots are taken, which corresponded with a slow reduction in the voltage-holding ability of the HGI.

In particular, the part of the flashover indicated by the arrows appears to increase in length as the test sequence progresses. Again, the flashover image corresponds with damage seen on the HGI surface (Fig. 9). The location indicated by the arrows in Figs. 7 and 9 also corresponded to a stripe of white phosphor paint applied to the cathode in an unsuccessful attempt to observe the secondary electron avalanche. This paint formed a cathode triple junction, and an apparent voltage enhancement. However, Figs. 8 and 9 show that other regions of the HGI received similar damage despite not being located near the phosphor paint.

Another aim of our experiments is to perform direct comparisons between HGIs designed according to the two theories presented in Section 2. The tests described above were all carried out using HGIs with metal layers that were twenty to one-hundred times thinner than the dielectric layers. An initial test was also carried out with an HGI having metal layers and dielectric layers of approximately the same thickness (Fig. 10). The maximum gradient supported by this

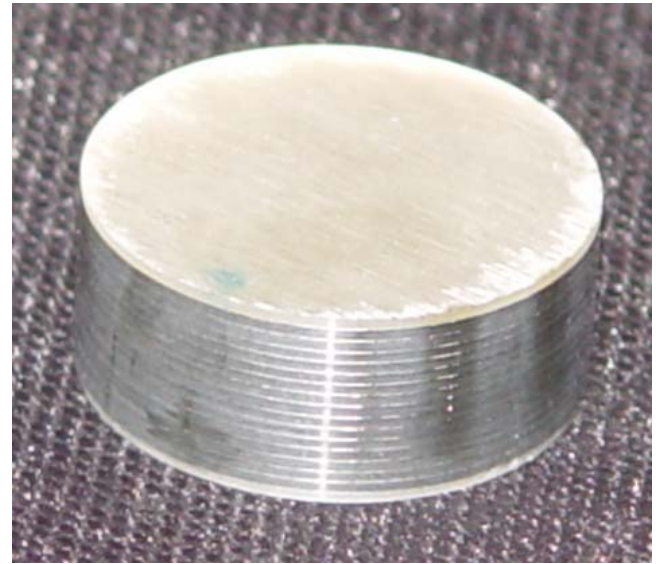

Fig. 8. HGI "R171," after being subjected to 223 surface flashover events, showing accumulated damage. HGI diameter is $2.54 \mathrm{~cm}$ and height is 1.015 $\mathrm{cm}$ [27].

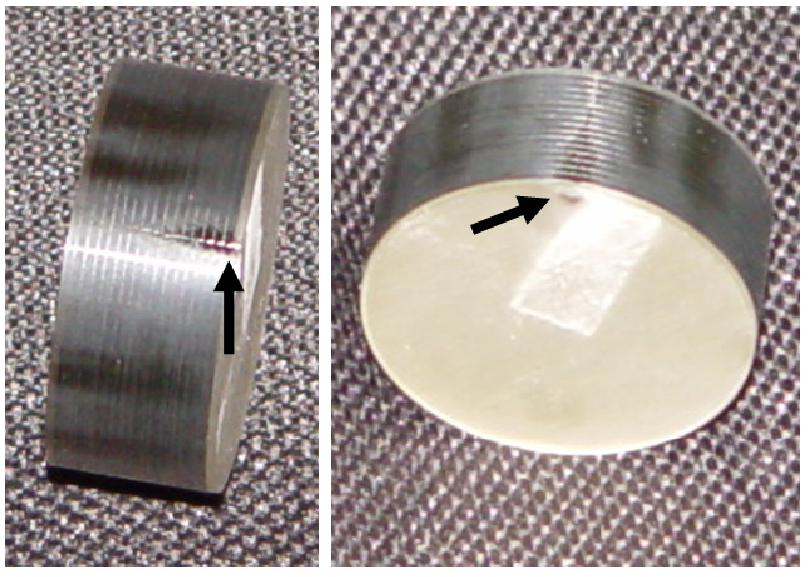

Fig. 9. HGI "R171." The location indicated by the arrows in this figure correspond to the location indicated by the arrow in Fig. 5B. The white rectangle on the bottom of the HGI is phosphor paint transferred from the cathode.

HGI without flashover was $10.0 \mathrm{MV} / \mathrm{m}$, which was worse than a thin-metal HGI ( $>21.1 \mathrm{MV} / \mathrm{m})$ and pure Rexolite slug $(16.6$

$\mathrm{MV} / \mathrm{m}$ ) having equal diameter and approximately equal height. However, the thick-metal HGI shown in Fig. 10 was not a proper test of the optimized Leopold theory, since its initial layer was a full thickness of dielectric rather than the half-thickness of metal specified in [24]. Surface flashover patterns of the thick-metal HGI were similar to those of the thin-metal HGIs (Fig. 11).

In spite of the damage mechanisms described here, in no HGI testing did we observe a sudden collapse in the voltageholding ability of an HGI, as is sometimes seen with conventional insulators subjected to surface flashover. Rather, our tests suggest a slow degradation in the insulator 
strength as damage accumulates. This may be due to a compartmentation effect provided by the alternating layers used in HGIs.

Our tests have shown that HGI surface flashover events evolve very quickly. To date, all flashover events observed in small sample tests have occurred on the leading edge of the applied voltage waveform. In these cases, the flashover

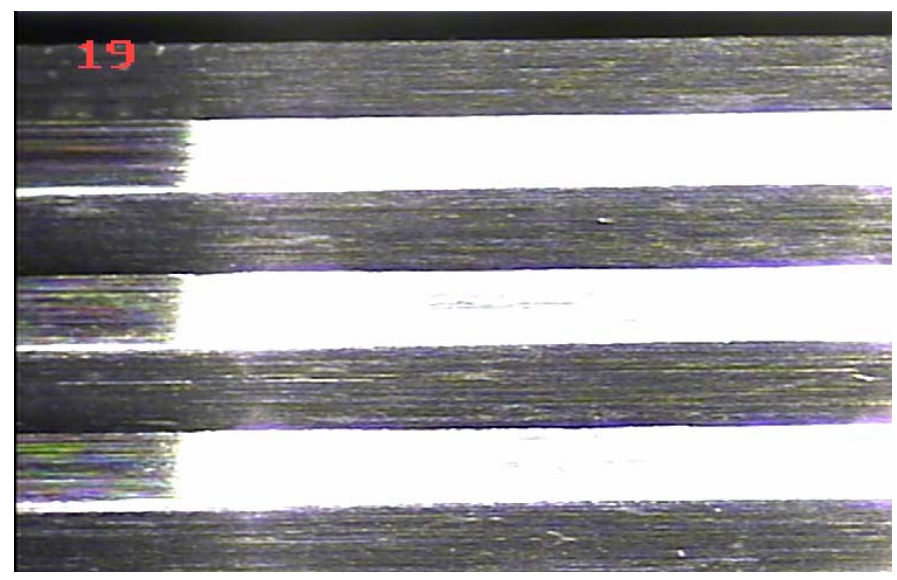

Fig. 10. HGI "R011." Microphotograph of a thick-metal HGI, showing initial full layer of dielectric at top of image. Metal layers are $0.305 \mathrm{~mm}$ thick and Rexolite layers are $0.254 \mathrm{~mm}$ thick.

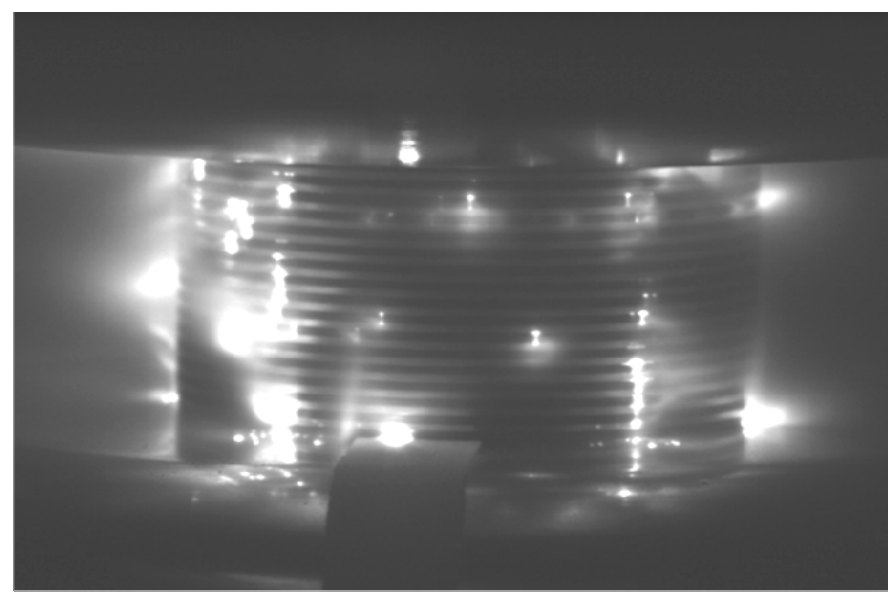

Fig. 11. HGI "R011." Thick-metal HGI undergoing representative surface flashover event. Sample height is $12.36 \mathrm{~mm}$.

prevents the Marx from fully erecting, as shown in Fig. 2. During normal operation, the peak voltage occurs approximately $25 \mathrm{~ns}$ into the pulse; during flashover events, it occurs between 5 ns and 17 ns into the pulse. Comparison of open-shutter and gated camera images indicates that flashover events take on their final shapes in less than $30 \mathrm{~ns}$.

\subsection{Large Sample Tests}

For larger HGI samples, we are using a modified Pulserad 110A originally built for flash radiography. It consists of a 10-stage Marx charging a $40 \Omega$ pulse forming line, and was designed to deliver a $20 \mathrm{~ns}, 1.2 \mathrm{MV}$ pulse into a matched load.
We modified the output region of the Pulserad to apply voltage across a larger HGI sample in an attempt to learn whether empirical length and area scaling rules derived from conventional insulator tests are applicable to HGIs. A series resistor was added to limit energy delivered to the test sample to less than $100 \mathrm{~J}$ in the event of surface flashover. The output of the Pulserad 110A was intentionally mismatched to produce a large voltage reversal across the test part to study the effect

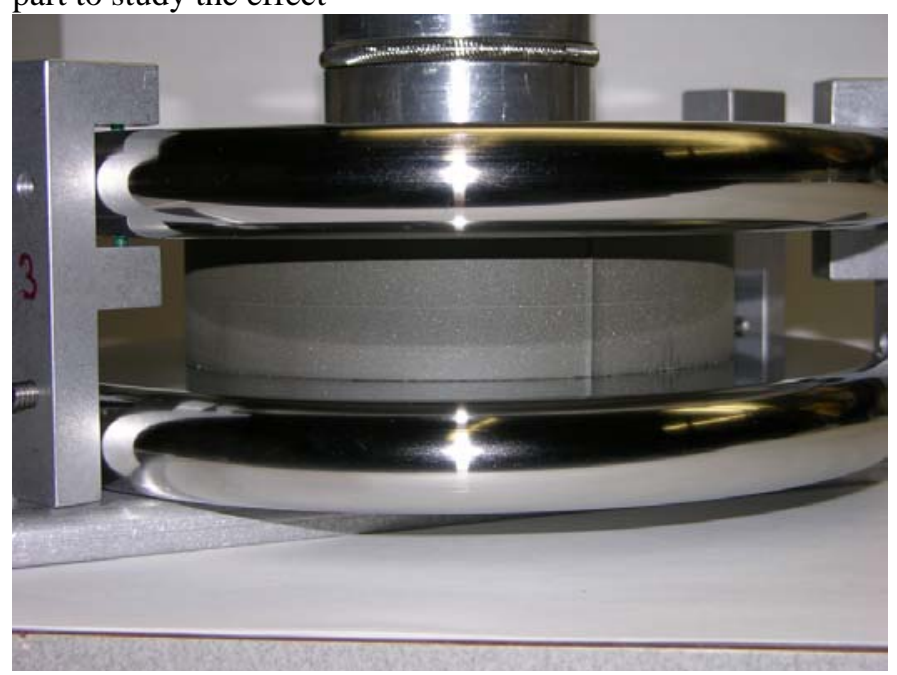

Fig. 12. Large HGI sample in test fixture.

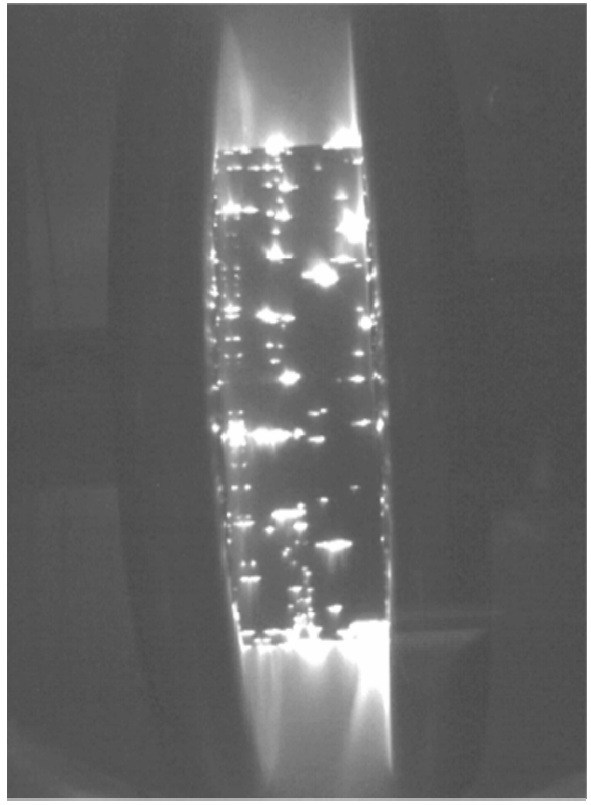

Fig. 13. Large HGI sample undergoing surface flashover.

of voltage waveform on insulator strength. Our first sample part was an unpolished, water-jet cut HGI fabricated from Kapton and stainless steel (Fig. 12). The sample had a diameter of $150 \mathrm{~mm}$ and a height of $35 \mathrm{~mm}$, which gives roughly 3.5 times the length and 20 times the surface area in comparison with the smaller samples. This part failed at 550 $\mathrm{kV}$, at a gradient of just under $16 \mathrm{MV} / \mathrm{m}$ (Fig. 13). First 
indications are that performance of the larger sample is in line with the smaller test HGIs.

\section{CONCLUSIONS}

HGIs exhibit superior performance as high-voltage vacuum insulators, and as a result they are the leading candidates for a number of novel accelerator and pulsed power applications. At Lawrence Livermore, we are continuing to study HGIs in order to optimize their design and understand their ultimate limits. Recent tests have focused on studying the damage resulting from surface flashover events. These tests indicate that the damage type varies as a function of applied voltage gradient and that repeated flashovers at high gradient result in progressive damage, but that the HGI will continue to function (although with reduced strength) after a large number of flashovers. These results suggest that HGIs are well suited to high power, high voltage applications.

\section{ACKNOWLEDGMENT}

This work was performed under the auspices of the U.S. Department of Energy by University of California, Lawrence Livermore National Laboratory under Contract W-7405-Eng48.

\section{REFERENCES}

[1] This is an expanded version of a paper published in Proceedings of the 2006 International Power Modulator Conference, Washington, DC, 1418 May 2006.

[2] H. Boersch, H. Hamisch, and W. Ehrlich, "Oberflaechenentladung ueber Isolatoren im Vakuum," Zeitschrift fuer angewandte Physik 15, 518-525, 1963. Translated in NASA Technical Memorandum NASA TM-77936 (1985).

[3] A. Watson, "Pulsed Flashover in Vacuum," Journal of Applied Physics 38, 2019-2023, 1967.

[4] Y. Saito, "Vacuum System of High-Energy Accelerators: Electrical Breakdown in Vacuum," Revista Brasileira de Aplições d Vácuo 22, 3944 (2003).

[5] S.T. Pai and Q. Zhang, Introduction to High Power Pulse Technology, World Scientific: Singapore, 2003.

[6] E. Gray, personal communication.

[7] W.C. Nunnally, M. Krogh, C. Williams, F. Allen, D. Trimble, S. Sampayan, and G. Caporaso, "Investigation of vacuum insulator surface dielectric strength with nanosecond pulses," in Proc. 2003 Pulsed Power Conf., Dallas, TX, June 15-18, 2003. (UCRL-JC-151647)

[8] G.J. Caporaso, "New pulsed power technology for high current accelerators," in Proc. 2002 International Power Modulator Symposium, Hollywood, CA, June 30 - July 3, 2002.
[9] G.J. Caporaso, "Progress in Induction Linacs," in Proc. 20th International Linear Accelerator Conference, Monterey, CA, Aug. 2125, 2000. (UCRL-JC-138443)

[10] G.A. Westenskow, D.P. Grote, E. Halaxa, J.W. Kwan, and F. Bieniosek, "A Compact High-Brightness Heavy-Ion Injector," in Proc. 2005 Part. Accel. Conf., May 17-21, 2005. (UCRL-CONF-212186)

[11] T.L. Houck, G.J. Caporaso, C.C. Shang, S.E. Sampayan, N.E. Molau, and M.L. Krogh, "Measured and Theoretical Characterization of the RF Properties of Stacked, High-Gradient Insulator Material," in Proc. 1997 Part. Accel. Conf., Vancouver, BC, May 12-16, 1997.

[12] J. McCarrick, Y.-J. Chen, T.L. Houck, and B.R. Poole, "Trapping Backstreaming Ions from an X-ray Converter Using an Induction Cell," in Proc. 19th International Linear Accelerator Conference, Chicago, IL, Aug. 23-28, 1998. (UCRL-JC-130421)

[13] T.L. Houck, G.A. Westenskow, J.-S. Kim, S. Eylon, E. Henestroza, S.S. $\mathrm{Yu}$, and D. Vanecek "Stacked Insulator Induction Accelerator Gaps," in Proc. 1997 Part. Accel. Conf., Vancouver, BC, May 12-16, 1997. (UCRL-JC-127273)

[14] S. Sampayan, G. Caporaso, B. Carder, M. Norton, D. Trimble, and J. Elizondo, "Optically Induced Surface Flashover Switching for the Dielectric Wall Accelerator," in Proc. 1995 Part. Accel. Conf.

[15] S. Sampayan, G. Caporaso, B. Carder, Y. Chen, C. Holmes, E. Lauer, D. Trimble, J. Elizondo, M. Krogh, B. Rosenblum, C. Eichenberger, and J. Fockler, "High Gradient Insulator Technology for the Dielectric Wall Accelerator," in Proc. 1995 Part. Accel. Conf.

[16] J.F. McCarrick, G.J. Caporaso, and Y.-J. Chen, "Beam Transport in a Compact Dielectric Wall Induction Accelerator System for Pulsed Radiography," in Proc. 2005 Part. Accel. Conf., Knoxville, TN, May 1620, 2005. (UCRL-CONF-212165)

[17] S. Sampayan, et al., in Proc. 2006 International Power Modulator Conference, Washington, DC, 14-18 May 2006.

[18] M. Rhodes et al., in Proc. 2006 International Power Modulator Conference, Washington, DC, 14-18 May 2006.

[19] I. Smith, personal communication, 26 May 2006.

[20] J.M. Elizondo, "Novel High Voltage Vacuum Surface Flashover Insulator Technology," in Proc. 1993 Pulsed Power Conf., Albuquerque, NM, June 21-23, 1993.

[21] J.M. Elizondo, A. Dragt, M. Krogh, D. Brooks, R. Smelser, P. Aragon, H. Happek, D. Torres, K.D. Ware, and K.R. Prestwich, "Vacuum flashover characteristics of laminated polystyrene insulators," in Proc. 1999 Pulsed Power Conf., Monterey, CA, May 27-30, 1999.

[22] M. Krogh, personal communication (2006).

[23] W.R. Cravey, G.L. Devlin, C.S. Mayberry, and J.N. Downing, "Investigation of a high voltage vacuum insulator for the DARHT accelerator," in Proc. 1997 Pulsed Power Conf.

[24] J.G. Leopold, U. Dai, Y. Finkelstein, E. Weissman, and S. Humphries, "Optimizing the Performance of Flat-surface, High-gradient Vacuum Insulators," IEEE Trans. Dielectrics Elect. Insul. 12, 530-536, June 2005.

[25] J.R. Pierce, Theory and Design of Electron Beams, New York: Van Nostrand, 1954.

[26] M. Reiser, Theory and Design of Charged Particle Beams, J. Wiley: New York, 1994.

[27] J.R. Harris, "Multilayer High-Gradient Insulators," in Proc. XXII International Symposium on Discharges and Electrical Insulation in Vacuum, Matsue, Japan, 25-29 Sept. 2006. 